\title{
Functional connectivity changes and their relationship with clinical disability and white matter integrity in patients with relapsing-remitting multiple sclerosis
}

Multiple Sclerosis Journal

$1-12$

DOI: $10.1177 /$

1352458514568826

(C) The Author(s), 2015. Reprints and permissions http://www.sagepub.co.uk/ journalsPermissions.nav

\author{
Emilia Sbardella, F Tona, N Petsas, N Upadhyay, MC Piattella, N Filippini, L Prosperini, \\ C Pozzilli and P Pantano
}

\begin{abstract}
Background and objective: To define the pathological substrate underlying disability in multiple sclerosis by evaluating the relationship of resting-state functional connectivity with microstructural brain damage, as assessed by diffusion tensor imaging, and clinical impairments.

Methods: Thirty relapsing-remitting patients and 24 controls underwent 3T-MRI; motor abilities were evaluated by using measures of walking speed, hand dexterity and balance capability, while information processing speed was evaluated by a paced auditory serial addiction task. Independent component analysis and tract-based spatial statistics were applied to RS-fMRI and diffusion tensor imaging data using FSL software. Group differences, after dual regression, and clinical correlations were modelled with GeneralLinear-Model and corrected for multiple comparisons.

Results: Patients showed decreased functional connectivity in 5 of 11 resting-state-networks (cerebellar, executive-control, medial-visual, basal ganglia and sensorimotor), changes in inter-network correlations and widespread white matter microstructural damage. In multiple sclerosis, corpus callosum microstructural damage positively correlated with functional connectivity in cerebellar and auditory networks. Moreover, functional connectivity within the medial-visual network inversely correlated with information processing speed. White matter widespread microstructural damage inversely correlated with both the paced auditory serial addiction task and hand dexterity.

Conclusions: Despite the within-network functional connectivity decrease and the widespread microstructural damage, the inter-network functional connectivity changes suggest a global brain functional rearrangement in multiple sclerosis. The correlation between functional connectivity alterations and callosal damage uncovers a link between functional and structural connectivity. Finally, functional connectivity abnormalities affect information processing speed rather than motor abilities.
\end{abstract}

Keywords: Multiple sclerosis, resting state, disability, functional connectivity, structural connectivity

Date received: 25 September 2014; revised: 11 November2014, 16 December 2014; accepted: 22

December 2014

\section{Introduction}

Advanced magnetic resonance imaging (MRI) techniques, such as functional (fMRI) and diffusion tensor imaging (DTI), have been applied in multiple sclerosis (MS) to identify functional and microstructural alterations that are not visible with conventional sequences. ${ }^{1,2}$
Specifically, resting state-fMRI (RS-fMRI) offers the opportunity to analyse the functional connectivity (FC) of different networks (RSNs), by detecting the coherence of the blood-oxygenation-level-dependent (BOLD)-signal. ${ }^{3}$ DTI evaluates white matter (WM) microarchitecture, by providing some metrics, i.e.
Correspondence to: Emilia Sbardella Department of Neurology and Psychiatry, Sapienza University, V.le dell’Università 30,00185 Rome, Italy. emilia.sbardella $a$ uniroma1.it Francesca Tona Nikolaos Petsas Neeraj Upadhyay Maria Cristina Piattella Luca Prosperini

Carlo Pozzilli Nicola Filippini Patrizia Pantano Department of Neurology and Psychiatry, University of Rome, Italy 
fractional anisotropy (FA), mean diffusivity (MD), and axial and radial diffusivity (AD, RD), which measure both myelin and axonal integrity. ${ }^{2,4}$ So far, although many RS analyses have been applied in MS, the meaning of FC changes as well as the correlation of FC abnormalities with structural damage and clinical impairment remain unclear. Diffuse RSNs abnormalities, indicating both increased ${ }^{5,6}$ and decreased ${ }^{7}$ connectivity, have been shown in patients, ${ }^{5-9}$ alterations of between-network connectivity have been also demonstrated, ${ }^{7,8,10}$ Nevertheless, whether FC changes reflect adaptive or maladaptive plasticity in pathological conditions is still debated ${ }^{1,6-10}$ Conversely, DTIderived results in MS are more consistent, with a widespread decrease in FA and increase in $\mathrm{MD}, \mathrm{AD}$ and RD having been detected throughout the $\mathrm{WM}^{2,11-13}$ However, studies investigating the relationship between functional and microstructural brain changes in MS provided conflicting results, according to which the increase in FC may be correlated either positively ${ }^{14}$ or negatively ${ }^{15,16}$ with structural damage. Finally, the correlation analyses of functional and structural changes with clinical impairment have only been focused on certain specific functions; ${ }^{6,15,16}$ moreover, while clinical deficits were clearly related to DTI abnormalities, ${ }^{2}$ discordant findings have been reported when the relationship between FC changes and disability was considered. $5,7-9$

In our work, we aimed at finding RSNs abnormalities in MS and at investigating their correlations with both microstructural damage and a large spectrum of clinical impairments. In this way, we pointed out a way of better defining the pathological substrate underlying clinical disability. For this purpose we have applied a data-driven whole-brain approach using RS-fMRI and DTI techniques in a cohort of relapsing-remitting MS subjects.

\section{Materials and methods}

\section{Participants}

We retrospectively studied 30 patients with a diagnosis of relapsing-remitting MS according to the revised McDonald criteria, ${ }^{17}$ aged between 18 to 50 years and an Expanded Disability Status Scale (EDSS) ${ }^{18}$ score of up to 4.0. The exclusion criteria were: concomitant neurological, otological and visual diseases, relapse and new medication over the previous six months, contraindications to MRI, presence of one or more gadolinium-enhancing lesions on basal MRI. Cognitive impairment, as detected by a Mini Mental State Examination (MMSE) score of less than 24, was an exclusion criterion, to avoid its influence on clinical scales. Nevertheless, no patient was excluded for having an MMSE score of less than 24. Patients underwent MRI and clinical evaluation, including EDSS and Multiple Sclerosis Functional Composite (MSFC) ${ }^{19}$ scales, to assess motor and cognitive functions. To more deeply analyse motor disability, we also considered posturography assessment, a measure of balance impairment. ${ }^{20}$ MSFC includes the Timed 25-Foot Walk (T25FW) for leg function/ambulation, the 9-Hole Peg Test (9HPT) for right and left arm/hand function and the Paced Auditory Serial Addition Test (PASAT) to assess attention, working memory and, in particular, information processing speed. The higher the T25FW and 9HPT scores, the lower the performance, the higher the PASAT score the higher the performance. The z-scores of the MSFC subscales were then calculated. ${ }^{19}$ None of the patients had previously performed MSFC testing. Balance ability was evaluated by a computer-based static posturography, and two quantitative measures, indicating in millimetres the balance stability with open and closed eyes, were obtained; the higher the values the lower the performance. ${ }^{20}$

Twenty-four matched healthy subjects (HS) constituted the control group; they underwent MRI, MSFC and posturography assessment. The Local Ethical Committee approved the study and written informed consent was obtained for all participants.

\section{MRI acquisition}

Imaging was performed with a Siemens Magnetom Verio 3 Tesla scanner (Siemens AG, Muenchen, DE). Slice orientation parallel to the sub-callosal line was assured by acquiring a multi-planar T1-weighted (T1-w) localizer at the beginning of each MRI acquisition.

All subjects underwent a multimodal MRI study, including:

1. RS-fMRI: BOLD single-shot echo-planar images $\mathrm{TR}=3000 \mathrm{~ms}, \mathrm{TE}=30 \mathrm{~ms}$, flip angle $=$ $89^{\circ}, 64 \times 64$ matrix, $\mathrm{FOV}=192^{2} \mathrm{~mm}, 50$ contiguous axial slices, $3 \mathrm{~mm}$ thick, 140 volumes, acquisition time $=7 \min 11 \mathrm{~s}$;

2. DTI: single-shot echo-planar spin-echo images, $\mathrm{TR}=12,200 \mathrm{~ms}, \mathrm{TE}=94 \mathrm{~ms}$, matrix $=96 \times 96$, $\mathrm{FOV}=250^{2} \mathrm{~mm}, 72$ contiguous axial slices, 2 $\mathrm{mm}$ thick, $b=0$ and $1000 \mathrm{~s} / \mathrm{mm}^{2}, 30$ gradient directions;

3. T1 volumetric imaging: 3D T1-w (T13D) magnetization-prepared rapid acquisition gradient echo (MPRAGE) sequence, TR $=1900 \mathrm{~ms}$, $\mathrm{TE}=2.93 \mathrm{~ms}$, flip angle $=9^{\circ}$, matrix $=$ 
$256 \times 256, \mathrm{FOV}=260^{2} \mathrm{~mm}, 176$ contiguous sagittal slices, $1 \mathrm{~mm}$ thick;

4. Dual turbo spin-echo, proton density and $\mathrm{T} 2-\mathrm{w}$ images: axial, $\mathrm{TR}=3320 \mathrm{~ms}, \mathrm{TE}=10 / 103 \mathrm{~ms}$, $384 \times 384$ matrix, FOV $=220^{2} \mathrm{~mm}, 25$ axial slices, $4 \mathrm{~mm}$ thick, $30 \%$ gap;

5. T1-w spin echo images after administration of gadolinium-based contrast agent, acquired only in patients: $\mathrm{TR}=550 \mathrm{~ms}, \mathrm{TE}=9.8 \mathrm{~ms}$, matrix $=320 \times 320, \mathrm{FOV}=240^{2} \mathrm{~mm}, 25$ axial slices, $4 \mathrm{~mm}$ thick, $30 \%$ gap.

\section{Image processing and data analysis}

All image analyses were carried out using FSL tools (FMRIB Software Library, http://www.fmrib.ox.ac. uk/fslwiki), version 5.0.7.

RS-fMRI analysis. Single-subject preprocessing was performed using FMRI Expert Analysis Tool (FEAT). Preprocessing included: (a) motion correction; (b) brain extraction; (c) spatial smoothing using a Gaussian kernel of full width at half maximum (FWHM) of $6 \mathrm{~mm}$; and (d) high pass temporal filtering using a cut-off of $150 \mathrm{~s}(0.007 \mathrm{~Hz})$. Functional data were aligned to structural images (within-subject) initially using linear registration and then optimized using a boundary-based registration approach. ${ }^{21}$ Structural images were transformed to standard space using a non-linear registration tool (FNIRT), and the resulting warp fields applied to the functional statistical summary images.

Differences in head motion were compared between patients and HS.

RSNs identification. Independent Component Analysis (ICA) of pre-processed RS data was carried out using Multivariate Exploratory Linear Optimized Decomposition into Independent Components (MELODIC). ${ }^{22}$ Because the automatic dimensionality estimation of the number of components to extract did not yield satisfactory results, the number of components was progressively increased and fixed to 40 , on the basis of the visual identification of the principal $\mathrm{RSNS}^{23,24}$ with the agreement of two scientists. Out of 40 components resulting from ICA analysis, 29 components were discarded, mainly because of reflected motion artefacts or BOLD signal drift. A total of 11 components were identified as RSNs: executive control (EC), sensorimotor (SM), right and left fronto-parietal (rFP, 1FP), default mode network (DMN), auditory, salience, basal ganglia $(\mathrm{BG})$, medial visual $(\mathrm{mV})$, lateral visual (IV) and cerebellar (CB). ${ }^{22,25}$
Between group RSNs comparison and RSNs clinical correlations. For each subject, a regression technique ('dual regression'), which allows for voxel-wise analysis, ${ }^{25}$ was applied to obtain individual RSN maps. General linear model (GLM) was applied using permutation-based non-parametric testing (5000 permutations) to assess between-group differences and to investigate the correlation between RSNs and clinical scores in patients. Significant clusters were determined by $\mathrm{Z}>2.3$ and then a family-wise-error (FWE) corrected cluster significance threshold of $p<0.05$ was applied to the suprathreshold clusters. Sex, age and normalized grey matter (NGM) were entered as covariates of no interests in the analyses. Anatomical localization of significant clusters was established by the Harvard-Oxford Structural Atlas (http://fsl.fmrib.ox.ac. $\mathrm{uk} / \mathrm{fs} \mathrm{f} / \mathrm{fs}$ lwiki/Atlases). In order to limit data analysis to voxels restrained within the 11 networks, we created a mask for each RSN that included only voxels surviving the threshold. Correlation analysis was performed on these masks only for the RSNs that differed between patients and controls. Correlations between these networks and clinical (9HPT, PASAT, T25FW and posturography assessment) as well as radiological variables, such as T2 lesion volume (LV), were performed by entering explanatory variables in the general linear modelling as implemented in FSL and then by running permutation analyses (randomize). Further, we created network masks including only those significant voxels differing between patients and controls and we correlated them with the clinical and radiological variables mentioned above by using the GLM.

Finally, parameter estimates, as expressed by Z-values in individual masked RS maps, were calculated to obtain a numerical value of the strength of RSNs temporal coherence.

All RS-fMRI results were further corrected for multiple comparisons, taking into account the number of variables by using Bonferroni method.

Inter-network correlations. To test for correlation between RSNs, we applied the FSLNets package implemented in MATLAB (Mathworks, Massachusetts, USA). ${ }^{26}$ The time courses of all maps in each subject were extracted and normalized by subjects' standard deviations, after correction for artefactual or no interest components; full correlation and partial correlation matrices of all RSNs' time courses were then created. Resulting Fisher's $r$ correlation coefficients were $z$ transformed and corrected for temporal autocorrelation.

$T$-values of correlations between each pair of RSNs, as well as differences in mean correlation values 
between patients and HS, were obtained after False Discovery Rate (FDR) correction. $P$ values $<0.05$ after Bonferroni correction were considered as significant.

\section{Structural MRI analysis}

TBSS and DTI parameters. 'Eddy current correction' was used to pre-process raw DTI images in order to correct for distortions due to the gradient directions applied. Subsequently, DTIFit was used to fit a diffusion tensor model at each voxel and generate individual FA, MD, AD and RD images.

FA maps were fed into the Tract-Based Spatial Statistic (TBSS) tool. The FA maps of all subjects were aligned into a common space by non-linear registration and averaged to obtain a mean FA skeleton. Finally, each subject's aligned FA data were projected onto this skeleton. Similar processes were applied to $\mathrm{MD}, \mathrm{AD}$ and $\mathrm{RD}$ maps using the individual registration and projection vectors obtained in the FA nonlinear registration and skeletonization stages. A voxel-wise cross-subject statistic analysis was then performed to compare MS patients and HS. GLM to assess differences in DTI metrics between patients and HS between-group differences, adjusted for subject's gender, age and brain volume, was applied to the spatial maps using permutation-based non-parametric testing (5000 permutations) with correction for multiple comparisons, by using a cluster-based correction approach and FWE-corrected $p$ value $<0.05$. GLM was also applied to identify the correlation between DTI metrics and both the RSNs parameter estimates of each network and the clinical scores: RSNs parameter estimates and clinical variables were entered in a correlation analysis as variables of interest, adjusted for the subjects' gender, age and LV. The resulting statistical maps threshold was set at $p<0.05$. TBSS results were further corrected taking into account the number of variables by using Bonferroni method. Significant WM tracts were localized by using FSL WM Atlas.

Lesion volume. LV was calculated on PD-w images using a semi-automated technique with the Jim 5.0 software (Xinapse System, Leicester, UK). Lesion filling was not applied.

Volumetric assessment. GM and WM volumes were obtained by using SienaX, part of FSL. First, the nonbrain tissue was stripped and then the brain and skull images were used to estimate the scaling between the subject's image and standard space. After segmentation, brain tissue volumes were calculated and multiplied by the estimated scaling factor, to reduce head-size-related variability between subjects and to obtain normalized volumes of GM, WM and global brain volume (GBV).

\section{Statistical analysis}

Statistical analysis was carried out using SPSS software, version 16.0 (SPSS, Chicago, USA). All values are reported as mean $\pm \mathrm{SD}$ or median and range, as appropriate. Demographic, behavioural and radiological differences between groups were tested using the $t$-test and the Exact Fisher's test, for continuous and categorical variables, respectively. Statistical threshold was set at $p \leqslant 0.05$. Pearson's correlation was applied to study the relationship between RS parameter estimates and LV and results were Bonferroni corrected.

\section{Results}

\section{Participants}

The demographic, clinical and radiological characteristics of the $30 \mathrm{MS}$ patients and $24 \mathrm{HS}$ are shown in Table 1. Patients performed worse than HS in all clinical measures.

\section{Resting state fMRI data}

No differences were found in head motion between patients and HS (absolute values 0.187 vs. 0.220 and relative values 0.033 vs. $0.036, p=0.201$ and $p=$ 0.528 , respectively).

Within- and between-network connectivity. By applying voxel-wise analysis, patients displayed reduced $\mathrm{FC}$ than HS within the following five RSNs: $\mathrm{CB}, \mathrm{EC}$, $\mathrm{mV}, \mathrm{BG}$ and SM (Figure 1). No areas of increased FC were detected in patients in comparison to HS. After Bonferroni correction across the RSNs employed in this study, no RSN was significantly different between the two groups. Table 2 shows the parameter estimates values calculated within voxels that significantly differed between patients and HS in five RSNs.

In both groups significant inter-network correlations and anti-correlations were found (Figure 2). In HS, significant correlations were detected between the DMN and both the IFP and auditory networks, between the $\mathrm{EC}$ and $\mathrm{BG}$ network, between the rFP and $\mathrm{CB}$, auditory and SM networks, and between salience and SM network. MS patients showed, besides all these correlations, additional significant correlations between auditory and SM networks and between 
Table 1. Demographic, behavioural and radiological characteristics in patients and healthy subjects.

\begin{tabular}{|c|c|c|c|}
\hline & Patients & HS & $p$ \\
\hline $\operatorname{Sex}(f / m)$ & $21 / 9$ & $14 / 10$ & 0.404 \\
\hline Age (y) & $35.0 \pm 8.0$ & $32.0 \pm 6.1$ & 0.133 \\
\hline Disease duration (y) & $10.1 \pm 6.2$ & NA & NA \\
\hline EDSS (median, range) & $2.5(0-4)$ & NA & NA \\
\hline PASAT & $28.97 \pm 11.96$ & $41.67 \pm 9.30$ & $<0.0001$ \\
\hline 9HPT r & $23.66 \pm 5.98$ & $15.62 \pm 1.69$ & 0.002 \\
\hline 9HРТ 1 & $26.02 \pm 9.05$ & $16.96 \pm 1.69$ & $<0.0001$ \\
\hline $25 \mathrm{FWT}$ & $9.08 \pm 5.45$ & $5.26 \pm 0.63$ & 0.004 \\
\hline Path_OE (mm) & $536 \pm 261$ & $221 \pm 58$ & $<0.0001$ \\
\hline Path_CE (mm) & $1060 \pm 398$ & $335 \pm 120$ & $<0.0001$ \\
\hline $\mathrm{LV}(\mathrm{mL})$ & $9.41 \pm 9.16$ & NA & NA \\
\hline $\operatorname{NGMV}\left(\mathrm{mm}^{3}\right)$ & $766,709.88 \pm 52,703.69$ & $810,924.36 \pm 45,107.70$ & 0.002 \\
\hline NWMV $\left(\mathrm{mm}^{3}\right)$ & $709,797.47 \pm 53,575.53$ & $764,214.23 \pm 37,707.12$ & $<0.0001$ \\
\hline $\operatorname{NGBV}\left(\mathrm{mm}^{3}\right)$ & $1,476,507.36 \pm 95,036.21$ & $1,575,138.59 \pm 74,355.64$ & $<0.0001$ \\
\hline FA & $0.45 \pm 0.042$ & $0.50 \pm 0.026$ & $<0.0001$ \\
\hline $\operatorname{MD}\left(\mathrm{mm}^{2} / \mathrm{s}\right)$ & $0.74 \times 10^{-3} \pm 5.30 \times 10^{-5}$ & $0.69 \times 10^{-3} \pm 2.15 \times 10^{-5}$ & $<0.0001$ \\
\hline $\mathrm{AD}\left(\mathrm{mm}^{2} / \mathrm{s}\right)$ & $1.30 \times 10^{-3} \pm 3.54 \times 10^{-5}$ & $1.20 \times 10^{-3} \pm 2.41 \times 10^{-5}$ & $<0.0001$ \\
\hline $\mathrm{RD}\left(\mathrm{mm}^{2} / \mathrm{s}\right)$ & $0.88 \times 10^{-3} \pm 4.63 \times 10^{-5}$ & $0.88 \times 10^{-3} \pm 2.02 \times 10^{-5}$ & 0.0008 \\
\hline \multicolumn{4}{|c|}{$\begin{array}{l}\text { Mean and SD are reported, otherwise indicated. A chi-square test was used to test difference in gender, whereas unpaired t-test was } \\
\text { used to test all other measures. Significant values are shown in bold. f: female; m: male; y: years; EDSS: Expanded Disability Statu } \\
\text { Scale; PASAT: Paced Auditory Serial Addiction Test; HPT r and l: hole peg test right and left; FWT: foot walk test; Path_OE: bal- } \\
\text { ance capability with open eyes; Path_CE: balance capability with closed eyes; LV: lesion volume; NGMV: normalized grey matter } \\
\text { volume; NWMV: normalized white matter volume; NGBV: normalized global brain volume; NA: not applicable. FA: fractional } \\
\text { anisotropy; MD: mean diffusivity; AD: axial diffusivity; RD: radial diffusivity. }\end{array}$} \\
\hline
\end{tabular}

rFP and lFP networks. In HS, significant anti-correlations were detected between IFP and both salience and EC networks. In patients, the former anti-correlation was preserved, while the latter was lost; patients also showed significant anti-correlations between DMN and both salience and IV networks and between IFP and BG networks. When the two groups of subjects were compared and results corrected for multiple comparison, patients demonstrated a weaker anti-correlation between 1FP and EC networks, a weaker correlation between auditory and BG networks and a stronger correlation between auditory and SM networks than HS.

\section{DTI data}

Patients, when compared to HS, displayed a significant reduction in FA values (Figure 3(a)) through the whole skeleton, mainly including the corpus callosum, anterior thalamic radiations, inferior and superior longitudinal fasciculi and cortico-spinal tracts. Results survived after brain volume correction and Bonferroni correction. No FA increase was found in patients. Patients also demonstrated significant increase in MD,
$\mathrm{AD}$ and $\mathrm{RD}$ values reflecting FA abnormalities distribution (data not shown).

\section{Correlations between fMRI and structural data}

When we correlated both RS parameter estimates, by using SPSS, and RSNs maps, by using TBSS, with disease burden, as assessed by T2 LV, no significance was found.

We also correlated microstructural damage, as measured by FA values, with parameter estimates of the 11 RSN maps by using TBSS. We found that the FA values in the anterior portion of the corpus callosum inversely correlated with temporal coherence within the $\mathrm{CB}$ and auditory networks (Figure $3(\mathrm{~d}, \mathrm{e})$ ). No direct correlation was found between FA and FC in any RSN.

\section{Correlations between MRI data and clinical impairment}

In patients, when the networks significantly different between patients and HS were correlated with clinical 

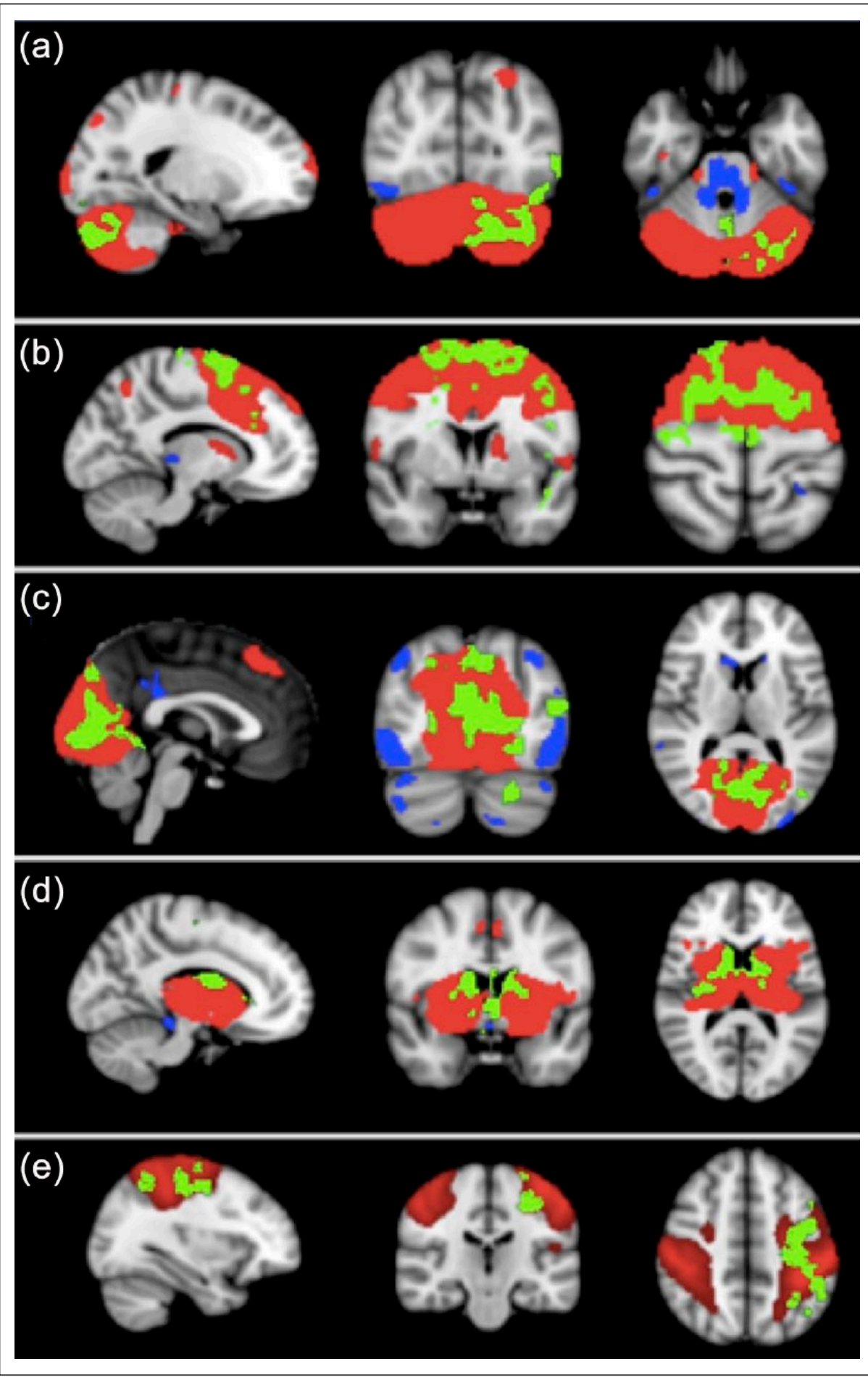

Figure 1. RSNs with decreased functional connectivity in 30 patients. In red positively correlated voxels, in blue negatively correlated voxels. In green, brain areas of decreased connectivity in patients after dual regression analysis within: (a) vermis, left crus I, left crus II, left occipital cortex of the cerebellar network; (b) superior frontal gyrus, paracingulate gyrus, precentral gyrus, anterior cingulate gyrus, bilaterally, and left insular cortex of the executive control network; (c) lingual gyrus, intracalcarine cortex, occipital pole, precuneus, bilaterally; left crus I and II of the medial visual network; (d) caudate bilaterally and right lenticular nucleus of the basal ganglia network; (e) left precentral gyrus and postcentral gyrus of the sensorimotor network.

Images are shown according to the radiological convention. fMRI results are overlaid on the MNI $1521 \mathrm{~mm}$ standard brain. Melodic, two-sample $t$-test, FWE corrected, $p<0.05$. 
Table 2. Values of parameter estimates within areas significantly different between patients and healthy subjects in five resting state networks.

\begin{tabular}{lccc}
\hline \multicolumn{1}{c}{ RSNs } & Patients & Healthy subjects & $p$ \\
\hline Cerebellar & $10.646 \pm 5.300$ & $20.230 \pm 7.897$ & $<\mathbf{0 . 0 0 0 1}$ \\
Executive control & $6.448 \pm 2.430$ & $13.914 \pm 5.029$ & $<\mathbf{0 . 0 0 0 1}$ \\
Medial visual & $19.119 \pm 6.139$ & $32.499 \pm 15.946$ & $<\mathbf{0 . 0 0 0 1}$ \\
Basal ganglia & $6.636 \pm 3.429$ & $15.035 \pm 5.243$ & $<\mathbf{0 . 0 0 0 1}$ \\
Sensorimotor & $11.732 \pm 2.943$ & $19.924 \pm 7.719$ & $<\mathbf{0 . 0 0 0 1}$ \\
\hline Values indicate parameter estimates, expressing strength of temporal coherence within clusters, and are expressed as mean and SD. \\
An unpaired $t$-test was used to assess between-group differences. Significant $p$ values are shown in bold. \\
\hline
\end{tabular}

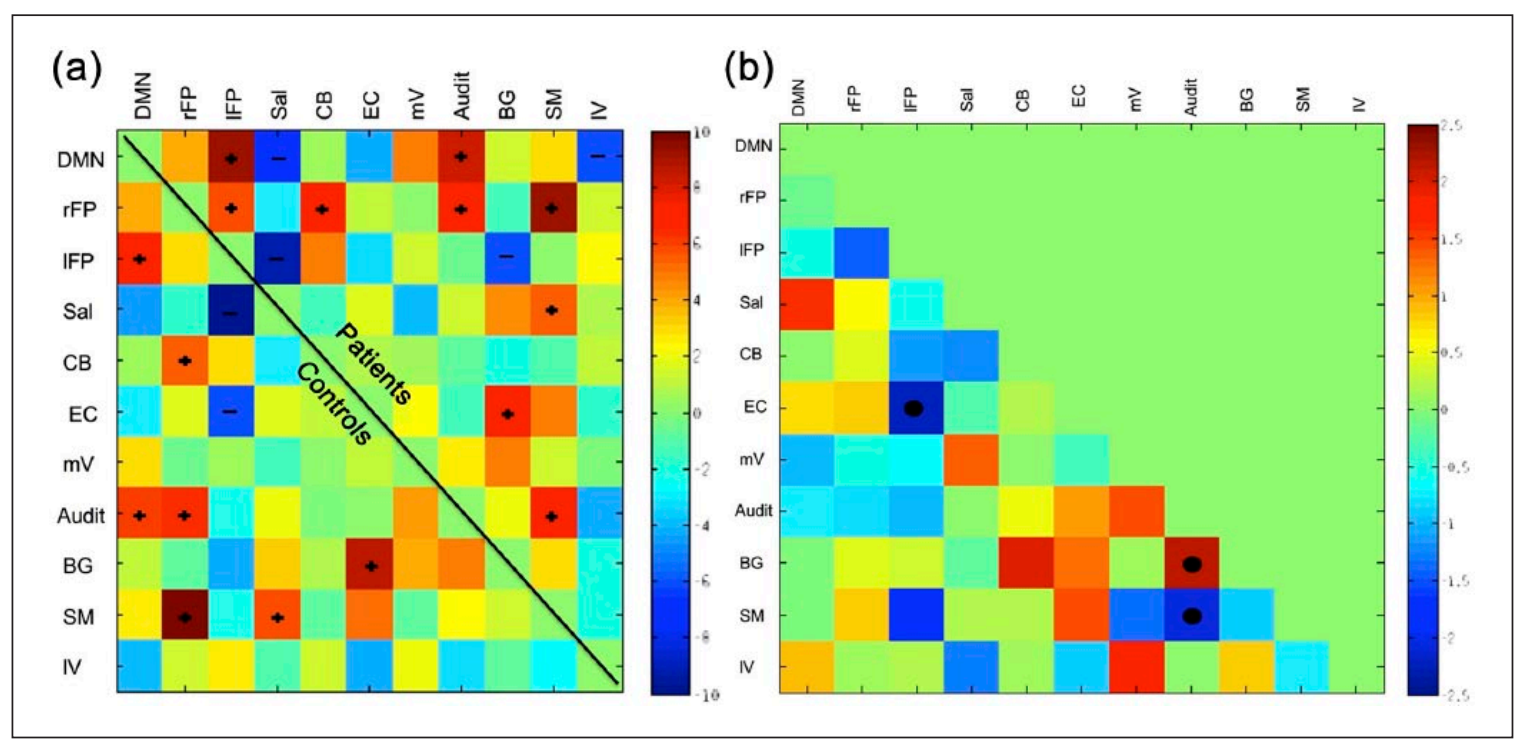

Figure 2. (a) Patients' and controls' $t$-values of between-network correlations. Plus signs: significant (positive) correlations; minus signs: significant anticorrelations. EC: executive control; SM: sensory-motor; 1FP-rFP: left and right fronto-parietal; DMN: posterior default mode network; BG: basal ganglia; $\mathrm{mV}$ : medial visual; CB: cerebellum; IV: lateral visual. Correlation analysis, FDR corrected, $p<0.004$ after Bonferroni correction. (b) Differences between patients and controls $t$-values in inter-network correlations. Blue squares: either a stronger between-network correlation or a weaker between-network anti-correlation in patients than in controls; red squares: either a stronger between-network anticorrelation or a weaker between-network correlation in patients than in controls. Dots: significant differences between the two groups. Patients demonstrated a weaker anti-correlation between left fronto-parietal and executive control networks, a weaker correlation between auditory and basal ganglia networks and a stronger correlation between auditory and sensorimotor networks than controls. Two-sample $t$-test, FDR corrected, $p<0.004$ after Bonferroni correction.

scores, using MELODIC, FC results significantly correlated with PASAT. In more detail, when the whole network was considered, we observed an inverse correlation between PASAT scores and EC as well as $\mathrm{mV}$ networks connectivity. However, when only voxels that were significantly different between patients and HS were considered, only $\mathrm{mV}$ networks maintained an inverse correlation with PASAT (Figure 4). Nevertheless, results did not survive to Bonferroni correction.

In patients, TBSS showed a significant positive correlation between FA values and clinical performance, as well as an inverse correlation between $\mathrm{MD}, \mathrm{AD}$ and RD and clinical performance. In particular, both left- and right-hand dexterity, as measured by $9 \mathrm{HPT}$ scores, and information processing speed, as measured by PASAT, correlated with FA values in most WM fibre tracts (Figure 3(b,c)). Almost the same areas were involved for $\mathrm{MD}, \mathrm{AD}$ and $\mathrm{RD}$ (data not shown). No inverse correlation between FA values and clinical performance was observed.

\section{Discussion}

We combined whole-brain RS-fMRI and DTI analysis to obtain complementary information on damage underlying clinical impairment in MS, by focusing on 



Figure 3. TBSS results are shown in red and overlaid on MNI152 $1 \mathrm{~mm}$ standard brain. (a) Widespread areas of reduced FA in 30 patients with MS compared with 24 healthy subjects. Two-sample $t$-test, FWE corrected, $p<0.004$ after Bonferroni correction. (b) Areas of direct correlation between FA and PASAT. (c) Areas of direct correlation between FA and right 9HPT. Correlation analysis, FWE corrected, $p<0.008$ after Bonferroni correction.

$(\mathrm{d}, \mathrm{e})$ Areas in the corpus callosum showing an inverse correlation between FA and parameter estimates of cerebellum (d) and temporal (e) networks. Correlation analysis, FWE corrected, $p<0.004$ after Bonferroni correction. FA: fractional anisotropy, PASAT: paced auditory serial addiction test; 9HPT: 9 hole peg test. 


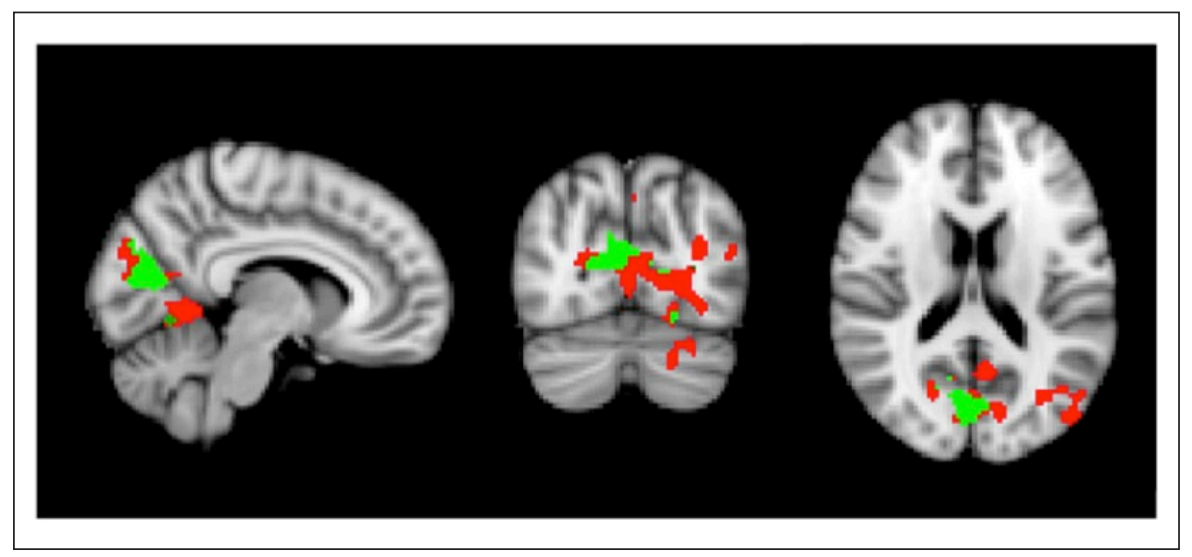

Figure 4. Inverse correlation between functional connectivity in the medial visual RSN (red) and PASAT performances in patients. Green indicates voxels of significant correlation located in bilateral supracalcarin cortices and right cuneal and intracalcarine cortices. Images are shown according to radiological conventions. fMRI results are overlaid on the MNI $1521 \mathrm{~mm}$ standard brain. Correlation analysis, FWE corrected, $p<0.05$.

a sample of RR patients to avoid the variability due to the inclusion of different MS phenotypes.

When RSNs were compared between patients and HS, taking into account differences in GM volume,, 27 five RSNs, i.e. cerebellar, executive control, medial visual, basal ganglia and sensorimotor, displayed decreased temporal coherence in MS. The FC reduction in patients has been previously reported ${ }^{1}$ and it was ascribed to several factors, i.e. diffuse brain hypometabolism, hypoperfusion, and the progressive accumulation of tissue damage in both lesions and in normally appearing brain matter. ${ }^{7,28}$ Nevertheless, FC has been demonstrated to be greater in many RSNs of patients at disease onset ${ }^{6}$ and with early RRMS $^{5}$ in comparison to controls. This suggests that brain reorganization, as expressed by changes in $\mathrm{FC}$, is probably a dynamical process across the disease course. Accordingly, the presence of an increase in temporal coherence of cerebral activity at the earliest stage of the disease probably acts as a compensatory phenomenon that may be lost in the more advanced phases of the disease, as a result of tissue damage progression. Unfortunately, we were probably not able to detect this phenomenon, as we studied subjects with heterogeneous disease duration, thus including patients at different stages of the disease.

In patients, we also found complex changes in betweennetwork interactions. Specifically, the neural anti-correlation between left fronto-parietal and executive control networks as well as the correlation between auditory and basal ganglia networks were weaker, while the correlation between auditory and sensorimotor networks was stronger in MS in comparison to HS.
Changes in global network function have been shown to depend on the underlying structural neuronal connections, but they have also been demonstrated as the response to transient focal disturbances without structural damage, such as reversible functional silencing after drug administration. ${ }^{29}$ Accordingly, our results might represent a global remodelling of functional large-scale connections, ${ }^{8,10,30}$ occurring as an attempt to contrast structural damage, in order to limit the clinical impact, as well as the result of temporary and not specific disease-induced molecular, neuronal or synaptic modifications.

In support of the former hypothesis, we found in our patients diffuse, significant, microstructural damage, as demonstrated by the reduction in the FA and the increase of $\mathrm{MD}, \mathrm{AD}$ and $\mathrm{RD}$ values throughout the skeleton. ${ }^{13,31}$ Moreover, a positive correlation between the microstructural damage in the anterior portion of the corpus callosum and the temporal coherence within the cerebellar and auditory networks was also detected. The finding of widespread microstructural damage is not surprising in MS, ${ }^{11-13,31}$ nor is the involvement of the corpus callosum, which is known to be one of the regions affected most over the disease course. ${ }^{32}$ The correlation between callosal fibre disruption and the temporal coherence within the cerebellar and auditory networks may be ascribed to the role played by the corpus callosum in many clinical functions, such as cognition, motor control and interhemispheric transfer of information, ${ }^{32}$ in which these networks are also involved. ${ }^{33,34}$ Since a reduction of temporal coherence within the cerebellar and auditory networks was detected in our population, we can only speculate that a more preserved FC concomitant with 
the presence of structural damage may represent a functional compensative attempt. Nevertheless, in patients, the auditory network demonstrated an increased inter-network connectivity, thus pointing to a likely relationship between the fibre connections' disruption and the FC changes in specific areas. Conversely, despite the widespread FA changes in the MS cohort, the correlation of microstructural connectivity with FC in only two networks may also suggest the partial independency of the functional and structural measures. Finally, despite our speculations, mostly because of the cross-sectional design of the study, we could not demonstrate a causal link between the two kinds of damage. Accordingly, whether functional changes represent only pathological modifications in connectivity remains to be proved.

With respect to HS, patients were clinically impaired, both in terms of motor capability and cognitive performances related to functions explored by PASAT. The 9HPT and PASAT scores were correlated with widespread microstructural alterations, as previously reported. ${ }^{27}$ On the other hand, when we investigated the relationships between clinical measures and FC of the whole networks that were abnormal in patients, differently from what we expected, i.e. an increased within-network synchronization to participate in adaptive mechanisms of neuroplasticity, ${ }^{6,35}$ we found an inverse correlation between PASAT performance and $\mathrm{FC}$ in the executive control and medial visual networks. While the executive control network is known to be implicated in several cognitive abilities, including information processing speed, which is measured by PASAT, 5,36 the involvement of the visual network is quite unexpected. Nevertheless, the visual network has recently been found to be disrupted in cognitively impaired patients. ${ }^{37}$ The inverse correlation between clinical performance and neural synchronization has recently been described and interpreted either as a consequence of maladaptive plasticity ${ }^{9}$ or as the expression of limited compensatory process. $^{5}$ However, when we restricted the analysis to those voxels significantly different between patients and HS, only the inverse correlation between PASAT and medial visual network was maintained. Thus, our results suggest that the clinical correlation with the executive control network, which is almost completely driven by normal areas, indicates physiological variations in connectivity related to PASAT performance. Conversely, the clinical correlation with abnormal voxels of medial visual areas is likely MS-specific and could indicate that the decreased connectivity is not a pathological marker, necessarily associated to worse performance, but could represent a part of the global rearrangement occurring during the disease course.

Surprisingly, we did not find any correlation between PASAT score and the Default Mode Network FC, as described in previous studies ${ }^{16,38,39}$ These discrepant results were likely due to differences in patients' characteristics, i.e. disease duration, phenotype and level of cognitive impairment between others' and our cohorts.

This study has certain limitations. Firstly, although our patients were consecutively selected and the disease duration varied considerably, we included patients with EDSS lower than 4, thus improving the homogeneity of our cohort. This criterion might have affected results of RSNs correlation with clinical scores, including the lack of correlation with EDSS, and limiting the generalization of the findings. Secondly, in the FC and DTI analyses, we did not take into account regional atrophy or focal lesion distribution, though we did consider the global GM involvement and regional WM microstructural damage. Thirdly, we explored cognitive capability by administering the PASAT alone, which is quite reliable in clinical assessments, ${ }^{36}$ even though it explores partial aspects of cognitive functions. Fourthly, we only considered FA values for correlation between microstructural damage and FC, but a strict correspondence between the various DTI parameters has emerged in our study, also confirming previous results. ${ }^{27}$ Finally, the cross-sectional design prevents any definitive conclusion about the interpretation of FC changes, and longitudinal studies are warranted.

\section{Conclusions}

This study demonstrates widespread structural and functional abnormalities in MS patients and a close relationship between callosal structural disruption and neural synchronization in some RSNs. The within-network FC correlation with clinical impairment is not yet clear and may be interpreted as maladaptive plasticity, an ineffective compensation mechanism or even a physiological mechanism in specific networks. Nevertheless, between-network functional changes point to a global functional rearrangement, likely as an attempt to limit the impact of structural damage on clinical manifestations in MS.

\section{Conflict of interest}

None declared. 


\section{Funding}

This work was partially supported by FISM (grant number 2012/R/12).

\section{References}

1. Filippi M, Agosta F, Spinelli EG, et al. Imaging resting state brain function in multiple sclerosis. $J$ Neurol 2013; 260: 1709-1713.

2. Sbardella E, Tona F, Petsas N, et al. DTI measurements in multiple sclerosis: Evaluation of brain damage and clinical implications. Mult Scler Int 2013; 2013: 671730 .

3. Fox MD and Raichle ME. Spontaneous fluctuations in brain activity observed with functional magnetic resonance imaging. Nat Rev Neurosci 2007; 8: $700-711$.

4. Basser PJ and Jones DK. Diffusion-tensor MRI: Theory, experimental design and data analysis $-\mathrm{A}$ technical review. NMR Biomed 2002; 15: 456-467.

5. Faivre A, Rico A, Zaaraoui W, et al. Assessing brain connectivity at rest is clinically relevant in early multiple sclerosis. Mult Scler 2012; 18: 1251-1258.

6. Roosendaal SD, Schoonheim MM, Hulst HE, et al. Resting state networks change in clinically isolated syndrome. Brain J Neurol 2010; 133: 1612-1621.

7. Rocca MA, Valsasina P, Martinelli V, et al. Largescale neuronal network dysfunction in relapsingremitting multiple sclerosis. Neurology 2012; 79: 1449-1457.

8. Hawellek DJ, Hipp JF, Lewis CM, et al. Increased functional connectivity indicates the severity of cognitive impairment in multiple sclerosis. Proc Natl Acad Sci USA 2011; 108: 19066-19071.

9. Tona F, Petsas N, Sbardella E, et al. Multiple sclerosis: Altered thalamic resting-state functional connectivity and its effect on cognitive function. Radiology 2014; 271: 814-821.

10. Gamboa OL, Tagliazucchi E, von Wegner F, et al. Working memory performance of early MS patients correlates inversely with modularity increases in resting state functional connectivity networks. NeuroImage 2014; 94C: 385-395.

11. Cercignani M, Iannucci G, Rocca MA, et al. Pathologic damage in MS assessed by diffusionweighted and magnetization transfer MRI. Neurology 2000; 54: 1139-1144.

12. Ciccarelli O, Werring DJ, Wheeler-Kingshott CA, et al. Investigation of MS normal-appearing brain using diffusion tensor MRI with clinical correlations. Neurology 2001; 56: 926-933.
13. Raz E, Cercignani M, Sbardella E, et al. Clinically isolated syndrome suggestive of multiple sclerosis: Voxelwise regional investigation of white and gray matter. Radiology 2010; 254: 227-234.

14. Roosendaal SD, Hulst HE, Vrenken H, et al. Structural and functional hippocampal changes in multiple sclerosis patients with intact memory function. Radiology 2010; 255: 595-604.

15. Lowe MJ, Beall EB, Sakaie KE, et al. Resting state sensorimotor functional connectivity in multiple sclerosis inversely correlates with transcallosal motor pathway transverse diffusivity. Hum Brain Mapp 2008; 29: 818-827.

16. Louapre C, Perlbarg V, García-Lorenzo D, et al. Brain networks disconnection in early multiple sclerosis cognitive deficits: An anatomofunctional study. Hum Brain Mapp 2014; 35(9):4706-17.

17. Polman CH, Reingold SC, Edan G, et al. Diagnostic criteria for multiple sclerosis: 2005 revisions to the 'McDonald Criteria'. Ann Neurol 2005; 58: 840-846.

18. Kurtzke JF. Rating neurologic impairment in multiple sclerosis: An expanded disability status scale (EDSS). Neurology 1983; 33: 1444-1452.

19. Uitdehaag BMJ, Adèr HJ, Roosma TJA, et al. Multiple sclerosis functional composite: Impact of reference population and interpretation of changes. Mult Scler 2002; 8: 366-371.

20. Prosperini L, Sbardella E, Raz E, et al. Multiple sclerosis: White and gray matter damage associated with balance deficit detected at static posturography. Radiology 2013; 268: 181-189.

21. Greve DN and Fischl B. Accurate and robust brain image alignment using boundary-based registration. NeuroImage 2009; 48: 63-72.

22. Beckmann CF, DeLuca M, Devlin JT, et al. Investigations into resting-state connectivity using independent component analysis. Philos T Roy Soc B 2005; 360: 1001-1013.

23. Smith SM, Fox PT, Miller KL, et al. Correspondence of the brain's functional architecture during activation and rest. Proc Natl Acad Sci USA 2009; 106: 1304013045.

24. Menon V and Uddin LQ. Saliency, switching, attention and control: A network model of insula function. Brain Struct Funct 2010; 214: 655-667.

25. Filippini N, Nickerson LD, Beckmann CF, et al. Agerelated adaptations of brain function during a memory task are also present at rest. NeuroImage 2012; 59: 3821-3828.

26. Smith SM, Beckmann CF, Andersson J, et al. Resting-state fMRI in the Human Connectome Project. NeuroImage 2013; 80: 144-168. 
Visit SAGE journals online http://msj.sagepub.com

@ SAGE journals
27. Sbardella E, Petsas N, Tona F, et al. Assessing the correlation between grey and white matter damage with motor and cognitive impairment in multiple sclerosis patients. PloS One 2013; 8: e63250.

28. Bakshi R, Miletich RS, Kinkel PR, et al. Highresolution fluorodeoxyglucose positron emission tomography shows both global and regional cerebral hypometabolism in multiple sclerosis. $J$ Neuroimaging 1998; 8: 228-234.

29. Otte WM, van der Marel K, Braun KP, et al. Effects of transient unilateral functional brain disruption on global neural network status in rats: a methods paper. Front Syst Neurosci 2014; 8: 40.

30. Hardmeier M, Schoonheim MM, Geurts JJG, et al. Cognitive dysfunction in early multiple sclerosis: Altered centrality derived from restingstate functional connectivity using magnetoencephalography. PloS One 2012; 7: e42087.

31. Sbardella E, Tomassini V, Stromillo M, et al. Pronounced focal and diffuse brain damage predicts short-term disease evolution in patients with clinically isolated syndrome suggestive of multiple sclerosis. Mult Scler 2011; 17: 1432-1440.

32. Ternes A-M, Maller JJ, Fielding J, et al. Investigating the role of the corpus callosum in regulating motor overflow in multiple sclerosis. $J$ Neurol 2013; 260: 1997-2004.

33. Reeber SL, Otis TS and Sillitoe RV. New roles for the cerebellum in health and disease. Front Syst Neurosci 2013; 7: 83 .

34. Formisano R, Schmidhuber-Eiler B, Saltuari L, et al. Neuropsychological outcome after traumatic temporal lobe damage. Acta Neurochir 1991; 109: 1-4.

35. Liu Y, Liang P, Duan Y, et al. Brain plasticity in relapsing-remitting multiple sclerosis: Evidence from resting-state fMRI. J Neurol Sci 2011; 304: 127-131.

36. Forn C, Belenguer A, Belloch V, et al. Anatomical and functional differences between the paced auditory serial addition test and the symbol digit modalities test. J Clin Exp Neuropsychol 2011; 33: 42-50.

37. Adriaanse SM, Binnewijzend MAA, Ossenkoppele $\mathrm{R}$, et al. Widespread disruption of functional brain organization in early-onset Alzheimer's disease. PloS One 2014; 9: e102995.

38. Rocca MA, Valsasina P, Absinta M, et al. Defaultmode network dysfunction and cognitive impairment in progressive MS. Neurology 2010; 74: 1252-1259.

39. Bonavita S, Gallo A, Sacco R, et al. Distributed changes in default-mode resting-state connectivity in multiple sclerosis. Mult Scler 2011; 17: 411-422. 